\title{
(2) OPEN ACCESS \\ Custom-made 3D-printed prosthesis and free vascularised fibula for humeral reconstruction after osteosarcoma resection in a 13-year-old patient
}

\author{
Giovanni Beltrami, ${ }^{1}$ Anna Maria Nucci, ${ }^{2}$ Angela Tamburini, ${ }^{3}$ Marco Innocenti ${ }^{4}$
}

\begin{abstract}
'Department of Pediatric Orthopedics and Pediatric Orthopedic Oncology, Azienda Ospedaliero Universitaria, Meyer Children Hospital, Firenze, Italy ${ }^{2}$ Department of Orthopedics and Traumatology, Azienda Ospedaliero Universitaria, Meyer Children Hospital, Florence, Italy ${ }^{3}$ Department of Pediatric Onco-Hematology, Azienda Ospedaliero Universitaria, Meyer Children Hospital, Firenze, Italy ${ }^{4}$ Department of Plastic Surgery and Microsurgery, Azienda Ospedaliero Universitaria Careggi, Firenze, Italy
\end{abstract}

Correspondence to Professor Giovanni Beltrami; gbeltrami663@gmail.com

Accepted 6 May 2021

Check for updates

(c) BMJ Publishing Group Limited 2021. Re-use permitted under CC BY-NC. No commercial re-use. See rights and permissions. Published by BMJ.

To cite: Beltrami $\mathrm{G}$, Nucci AM, Tamburini A et al. BMJ Case Rep

2021:14:e240726.

doi:10.1136/bcr-2020

240726

\section{SUMMARY}

Surgical reconstruction after humeral resection represents a challenging issue in orthopaedic oncology. Particularly in paediatric patients, the main concerns are maintaining limb function and reconstruction longevity. We describe a novel strategy of humeral reconstruction based on the use of a three-dimensional-printed custommade prosthesis in a 13-year-old patient diagnosed with osteosarcoma. The implant was specifically designed to sustain the native head, which was spared, as it was not involved by the neoplastic tissue. The mechanical support the prosthesis provided was associated with the biological stimulus of a free vascularised fibular graft to obtain an anatomic, functional and stable construct. This solution has had good longevity, and after 3 years of follow-up, the patient still shows excellent limb function and personal satisfaction.

\section{BACKGROUND}

The surgical management of malignant tumours in the humerus remains a debated issue, especially in the paediatric population. Recently, new chemotherapeutical approaches and advances in diagnostic imaging and surgical techniques have contributed to the growing success of limb-preserving surgery and improved patient survival rates. Today, orthopaedic oncology surgeons dispone of a huge range of surgical strategies (ie, prosthesis, prosthesis/ graft composites, massive allograft, recycled bone and autografts). ${ }^{1}$ However, treating large humeral sarcomas with involvement of the entire diaphysis is even more challenging. In order to maintain limb function while also performing a radical resection, any reconstruction strategy must be thoroughly planned. ${ }^{2}$ Here, we report the case of a 13 -year-old girl diagnosed with osteosarcoma involving the whole diaphysis of the left humerus treated with wide excision and reconstruction with a hollow three-dimensional (3D)-printed custom-made prosthesis filled with a free vascularised fibular graft (VFG), preserving the native humeral head.

\section{CASE PRESENTATION}

A 13-year-old right-handed female patient presented to our outpatient clinic with a history of left arm swelling and pain with intact forearm and hand function. She had no significant medical history. Left arm radiographs showed a nondisplaced diaphyseal pathological fracture of the humerus in the context of a complex bone lesion involving the entire diaphysis. Further diagnostic imaging was performed, including CT and MRI of the left arm and a CT of the chest. The MRI showed an intraosseous mass with a cystic aspect in the proximal and distal third of the humerus and a solid aspect in the middle third (figure 1). In consideration of the extension and heterogeneity of the neoplastic mass, four core needle biopsies were performed in different sites along the diaphysis. Histopathological analysis confirmed the diagnosis of osteoblastic osteosarcoma ABCB1/P-glycoprotein positive in only the middle part of the humeral diaphysis; the other specimens were negative. According to the Musculoskeletal Tumour Society (MTS) classification, the surgical stage was defined as IIA. ${ }^{3}$

\section{TREATMENT}

According to the ISG-Os2 protocol, the patient received neoadjuvant chemotherapy including cisplatin, adriamycin and methotrexate. Postchemotherapy imaging showed a stable disease associated with the reduction of the perilesional oedema and haematoma. In cooperation with industry engineers (Adler Ortho S.p.A., Cormano, Milano, Italy), a 3D reconstruction obtained from Digital Imaging and Communications in Medicine CT-imaging of the humerus, including the external shape and internal macrostructure, was designed with CAD/CAM technology. The titanium alloy implant was made employing electron beam additive manufacturing (EBM) technology (Adler Ortho). EBM is a relatively new technology for titanium powder sintering that facilitates the production of an implant of the exact shape of the $3 \mathrm{D}$ reconstruction. The technique employs a high-energy electron beam that melts the titanium powder in a vacuum chamber. The manufacturing process took 5 weeks to be completed.

Surgical intervention was performed as follows. Two surgical teams worked in parallel in order to reduce the duration of surgery: an oncology team performed the humerus resection, while a microsurgery team performed the contralateral fibula harvesting. A deltopectoral approach extended to the elbow was used for implant placement. A complete humeral resection was performed, with the only exception being the humeral head, as this was not invaded by neoplastic tissue. Taking advantage of the biological barrier provided by the growth plate, the osteotomy was performed just proximal to the metaphysis using a patient-specific disposable cutting block to obtain a nearly perfect bone-prosthesis match. The prosthesis comprised 


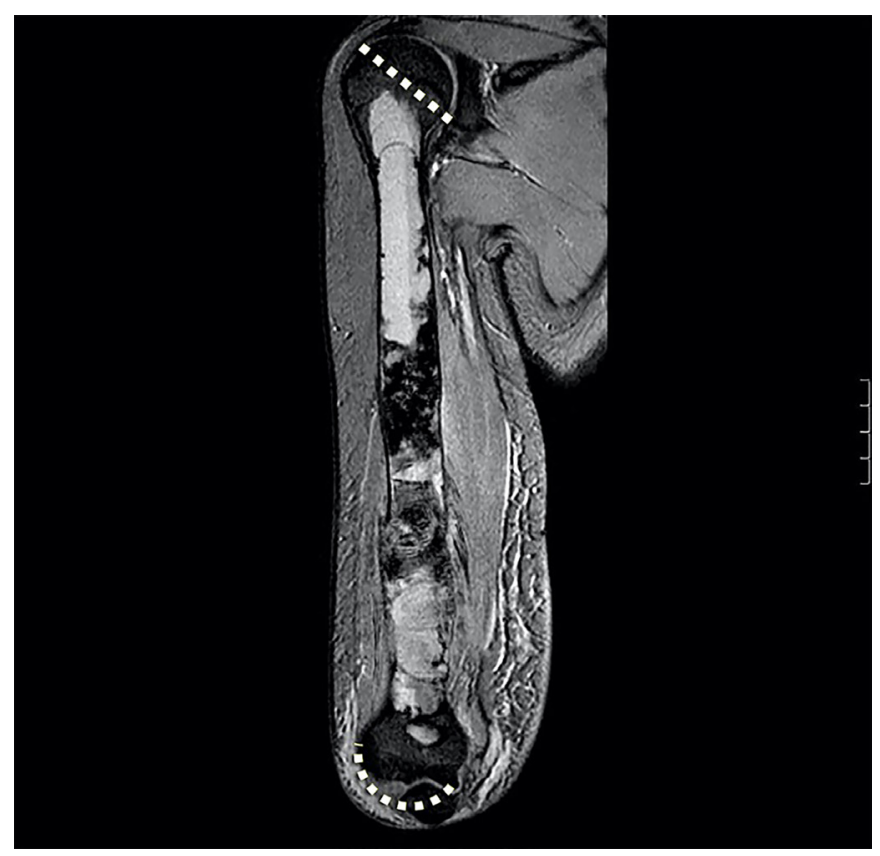

Figure 1 MRI showing an intraosseous mass with a cystic aspect in the proximal and distal third of the humerus and a solid aspect in the middle third. The dashed lines indicate intraoperative resection lines, illustrating that only the humeral head could be spared.

two separated parts bridged by two custom-made plates. The proximal prosthesis had a hollow design and was fixed to the humeral head by four screws located left subchondral (figure 2). In order to maintain head vascularisation, a VFG was harvested and placed inside the prosthetic body in direct contact with the humeral head. The VFG included microvascular anastomosis of the peroneal arteries and veins to the proximal recurrent branches of the humeral vessels. The residual empty space of the prosthesis was filled with cortico-cancellous bone chips. The rotator cuff was reinserted in dedicated holes of the prosthesis. The distal part of the prosthesis constituted an elbow hemiarthroplasty (EHA), sparing the intact ulnar and radial proximal epiphyses (figure 3). Elbow collateral ligaments were reinserted into the prosthetic medial and lateral epicondyles. Mersilene tapes were placed on both sides to further stabilise the elbow. The patient received intravenous prophylactic antibiotics perioperatively and oral antibiotics post-operatively for 6 weeks. The patient wore a sling for 4 weeks and began passive shoulder and elbow mobilisation 30 days after surgery-active movements were allowed 60 days after surgery. The histological analysis of the resected humerus confirmed the preoperative diagnosis and reported $80 \%$ necrosis and wide surgical margins. Adjuvant chemotherapy was administered according to ISG-Os2 protocol. In addition, the patient received a treatment with Mepact for 6 months.

\section{OUTCOME AND FOLLOW-UP}

No major complications occurred in the early and late postoperative stages. A superficial dehiscence of the surgical wound was observed in the early postoperative period, but healing was obtained after surgical wound revision. No donor site complications were observed. Currently, the patient is 3 years from surgery and free from local or distant disease progression. Physical examination demonstrates no upper limb discrepancy; shoulder passive abduction of $110^{\circ}$; flexion and extension of $90^{\circ}$ and $30^{\circ}$, respectively; and external and internal rotation of

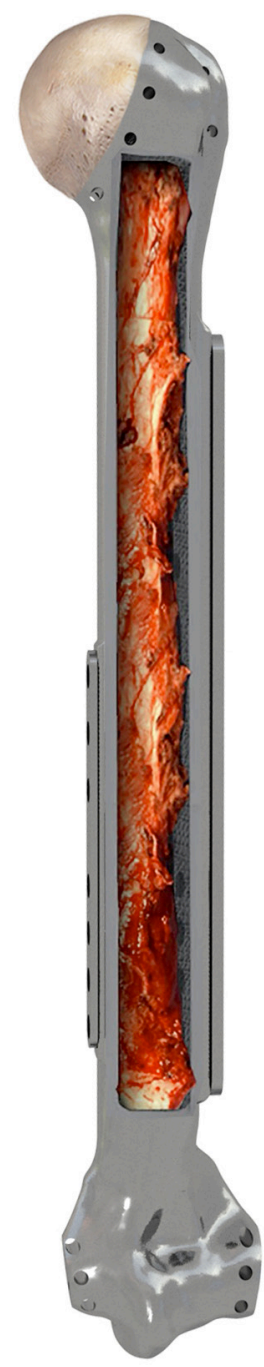

Figure 2 Implant rendering with the graft inside.

$45^{\circ}$ and $90^{\circ}$, respectively. The shoulder active range of motion (ROM) includes an abduction of $80^{\circ}$, flexion of $60^{\circ}$, extension of $30^{\circ}$, external rotation of $35^{\circ}$ and internal rotation of $90^{\circ}$ (figure 4). The elbow joint shows an extension lag of nearly $60^{\circ}$ and a maximal flexion of $130^{\circ}$. The elbow prono-supination is complete. The hand and wrist show complete functionality. There is complete recovery of sensory function. Three-year postoperative imaging shows the maintenance of a good quality of humeral head articular surface without signs of reabsorption (figure 5). The VFG appears enlarged in the proximal part of the prosthesis and partially integrated with the humeral head, and there is a partial reabsorption of the bone graft in the distal nonloading segment. At the moment, the patient does not complain of any pain and demonstrates only a slight limitation of activity in daily life. The MTS score for the upper limb is $26 / 30$.

\section{DISCUSSION}

Proximal humerus is a common site of bone sarcomas. The current treatment protocols in the field are based on limb salvage surgery and reconstruction strategies. Particularly in the paediatric population, the reconstructive project must respect bone growth potential and maintain limb function in the long term. With this purpose, many authors have proposed different reconstructive techniques such as arthroplasty, osteoarticular allografts, allograft-prosthesis composites, autologous grafts 


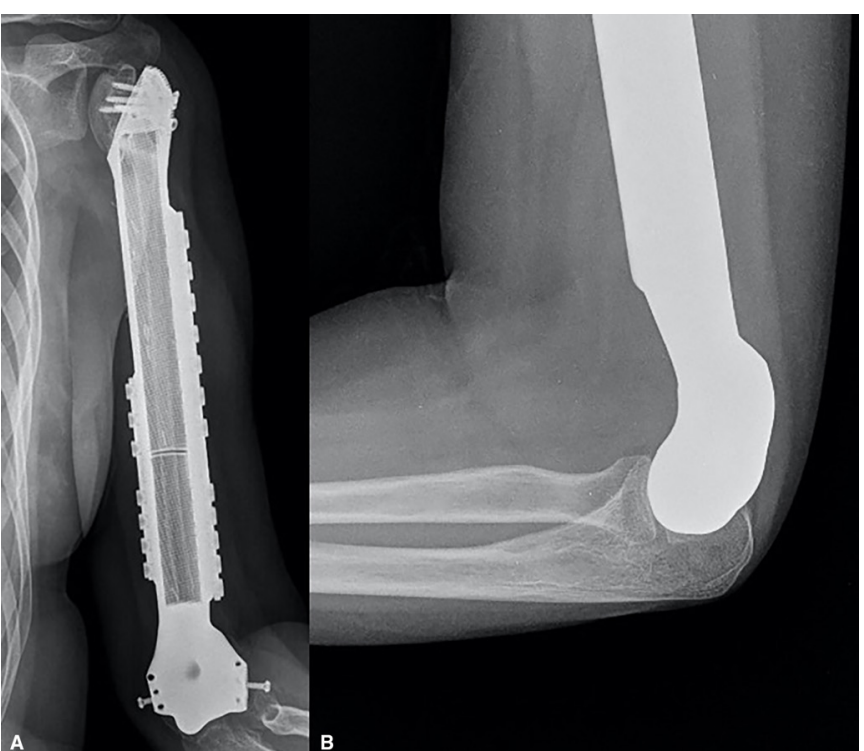

Figure 3 Radiographic follow-up. (A) Entire reconstruction. (B) Lateral view, osteoarticular elbow reconstruction.

associated with shoulder arthrodesis and recycled osteochondral grafts that are eventually associated with free VFG. ${ }^{145}$

When the neoplasia does not remain confined within the proximal humerus but involves a huge portion of diaphysis, the surgical reconstruction is even more complex and must be defined on a case-by-case basis. The uniqueness of the case reported here lies in the extended involvement of the humeral diaphysis associated with relative sparing of the humeral head. Taking into account these preoperative conditions, the available surgical options were as follows:

1. Total humeral mega-prosthesis replacement. This solution guarantees a good mechanical resistance, but it is generally accompanied by proximal migration of the implant and poor recovery of shoulder ROM with abduction that is rarely superior to $30^{\circ} .5$

2. Reverse shoulder arthroplasty (RSA). The role of RSA in oncological reconstruction is still contentious. This prosthetic can be implanted only in the presence of an active deltoid muscle and has the advantage of permitting better shoulder functionality. However, many studies in non-oncological patients reported a major complication rate compared with other types of arthroplasty. ${ }^{1}$ Finally, the longevity of RSA is still unknown.

3. Expandable prosthesis. In the case of paediatric bone sarcomas of the humerus, some authors have recently proposed this solution to accommodate lengthening in the growing child, but this technique implies further surgeries to obtain implant extension and is associated with high failure and complication rates. ${ }^{67}$ In addition, in our case, the patient's expected skeletal growth was limited, having been more than 2 years since her puberal spurt.

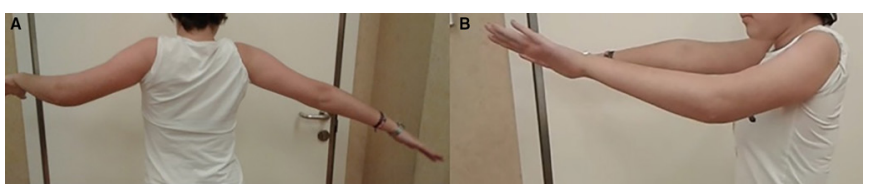

Figure 4 Clinical outcome, 3 years postoperatively. (A) Abduction. (B) Anteversion.

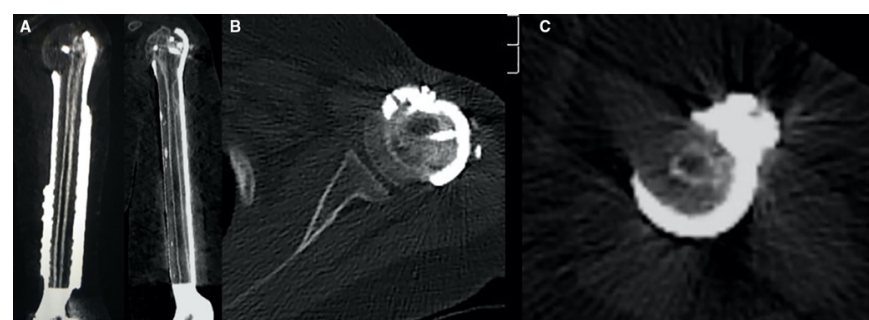

Figure 5 CT follow-up. (A) Entire reconstruction, 1-year postoperatively. (B) Entire reconstruction, 3 years postoperatively. (C) Humeral head, 3 years postoperatively. (D) Osseointegration between fibula bone graft and custom-made prosthesis.

4. Massive allograft. The use of tissue harvested from a cadaver with the possibility of biological attachment of ligaments and muscles could be a solution; however, especially in paediatric patients, an anatomical articular congruence is almost impossible to obtain, making the likelihood of early arthritis very high. Moreover, the allograft alone shows low mechanical resistance and poses a significant risk of resorption and fracture. ${ }^{8}$

5. Allograft-prosthesis composite. The association of an allograft with a prosthesis solves the problem of articular degeneration; however, a high risk of fracture and bone-graft non-union have been described. ${ }^{9}$

6. Recycled total humerus autograft. This is possible through pasteurisation, irradiation or freezing of the resected specimen and secondary replantation. These options allow for an anatomical reconstruction, but the result is fragile and prone to fracture. Another disadvantage is the impossibility of performing a complete histological analysis of the specimen to confirm the diagnosis. Furthermore, degeneration of the autograft cartilage could lead to early osteoarthritis. ${ }^{2}$ For this reason, some authors propose allograft composite prostheses of recycled bone. ${ }^{10}$

Considering the patient's age and functional demand, we adopted a different and innovative surgical plan aimed to preserve glenohumeral articulation. After complete humeral resection excepting the humeral epiphysis, the reconstructive project included mechanic support via a $3 \mathrm{D}$-printed custommade prosthesis and a biological stimulus provided by a VFG with the precise function of sustaining humeral head vascularisation. This surgical solution enabled a stable, functional and anatomical reconstruction of the glenohumeral joint and a cellular recolonisation of the humeral head due to the VFG.

Regarding elbow reconstruction, an EHA, rather than a total elbow arthroplasty (TEA), was realised. Compared with TEA, the major advantages of EHA in a young patient are preserving the ulnar bone stock and avoiding polyethylene particulate debris formation. Furthermore, a TEA implies the implantation of an ulnar stem-in paediatric patients, this poses a high risk of loosening and subsidence. ${ }^{11}$ In this case, we decided to reinsert the medial and lateral collateral ligament, adding Mersilene tapes to relatively limit articular excursion in favour of implant stability.

The adopted prosthesis was made of titanium alloy using electron beam melting technology. The implant consisted of two distinct parts, as the humerus measured about $35 \mathrm{~cm}$, and the $3 \mathrm{D}$-printer could only create a $20 \mathrm{~cm}$ sized piece. Two custommade plates were necessary to fasten the prosthetic components together. Obviously, the area of contact between the two parts is more exposed to bending forces and has a higher risk of plate breakage, but the biological support provided by the VFG should 
help to prevent this complication. However, an advantageous aspect of this particular configuration is the possibility of independent revision of the proximal and the distal humerus in the case of future development of secondary osteoarthritis.

To our knowledge, a similar surgical reconstruction strategy for humeral sarcoma has not previously been reported in the medical literature-ours represents the first case of humeral reconstruction with a custom-made prosthesis associated with VFG in a paediatric patient. In our opinion, 3D-printed prostheses could be a valuable new surgical instrument, especially when classical reconstruction techniques are not applicable. ${ }^{11} 12$ The association of a VFG seems a viable solution to sustain humeral head viability, avoid shoulder prosthetic replacement and preserve bone stock as much as possible. This reconstruction has demonstrated good durability: after 3 years of follow-up, the patient still shows excellent limb function and high personal satisfaction. This reconstructive strategy inevitably contains the risk of premature articular degeneration, the degree of which can only be defined with longer follow-up and further experience in applying the technique.

\section{Learning points}

- In the case of wide oncological humeral resection, the surgical strategy should be thoroughly planned to obtain a stable, long-lasting and functional reconstruction, especially in paediatric patients.

- The combination of three-dimensional-printed custom-made prostheses combined with vascularised fibular graft (VFG) is a viable option that can be tailored based on the features of neoplastic disease and considering a patient's functional demand.

- In our patient, we were able to maintain the native humeral head due to the association of mechanical support offered by the prosthesis and the biological stimulus provided by the VFG.

Contributors Conceptualisation, GB; methodology, GB; medical (oncological and surgical) expertise, GB, AMN, AT and Ml; writing-original draft preparation, GB; writing — review and editing, GB, AMN, AT and MI. All authors have read and agreed to the published version of the manuscript.

Funding The authors have not declared a specific grant for this research from any funding agency in the public, commercial or not-for-profit sectors.

Competing interests None declared.

Patient consent for publication Parental/guardian consent obtained.

Provenance and peer review Not commissioned; externally peer reviewed.

Open access This is an open access article distributed in accordance with the Creative Commons Attribution Non Commercial (CC BY-NC 4.0) license, which permits others to distribute, remix, adapt, build upon this work non-commercially, and license their derivative works on different terms, provided the original work is properly cited and the use is non-commercial. See: http://creativecommons.org/ licenses/by-nc/4.0/.

\section{REFERENCES}

1 Dubina A, Shiu B, Gilotra M, et al. What is the optimal reconstruction option after the resection of proximal humeral tumors? A systematic review. Open Orthop J 2017;11:203-11.

2 Wafa H, Reddy K, Grimer R, et al. Does total humeral endoprosthetic replacement provide reliable reconstruction with preservation of a useful extremity? Clin Orthop Relat Res 2015:473:917-25.

3 Enneking WF. A system of staging musculoskeletal neoplasms. Clin Orthop Relat Res 1986:204:9-24.

4 Muramatsu K, Fukano R, Ihara K, et al. Reconstruction of the proximal humerus by combined use of extracorporeally-irradiated osteochondral graft and free vascularized fibula following resection of Ewing sarcoma. J Plast Reconstr Aesthet Surg 2010;63:2177-80.

5 Puri A, Gulia A. The results of total humeral replacement following excision for primary bone tumour. J Bone Joint Surg Br 2012;94:1277-81.

6 Henderson ER, Gao J, Groundland J, et al. Expandable total humeral replacement in a child with osteosarcoma. Case Rep Orthop 2015;2015:1-5.

7 Tsuda Y, Tsoi K, Stevenson JD, et al. Extendable endoprostheses in skeletally immature patients: a study of 124 children surviving more than 10 years after resection of bone sarcomas. J Bone Joint Surg Am 2020;102:151-62.

8 Weber KL, Lin PP, Yasko AW. Complex segmental elbow reconstruction after tumor resection. Clin Orthop Relat Res 2003:415:31-44.

9 Ogink PT, Teunissen FR, Massier JR, et al. Allograft reconstruction of the humerus: complications and revision surgery. J Surg Oncol 2019;119:329-35.

10 Moran M, Stalley PD. Reconstruction of the proximal humerus with a composite of extracorporeally irradiated bone and endoprosthesis following excision of high grade primary bone sarcomas. Arch Orthop Trauma Surg 2009;129:1339-45.

11 Beltrami G. Custom 3D-printed finger proximal phalanx as salvage of limb function after aggressive recurrence of giant cell tumour. BMJ Case Rep 2018;5:bcr-2018226007

12 Beltrami G, Ristori G, Scoccianti G, et al. Latissimus dorsi rotational flap combined with a custom-made scapular prosthesis after oncological surgical resection: a report of two patients. BMC Cancer 2018;18:1003.

Copyright 2021 BMJ Publishing Group. All rights reserved. For permission to reuse any of this content visit

https://www.bmj.com/company/products-services/rights-and-licensing/permissions/

BMJ Case Report Fellows may re-use this article for personal use and teaching without any further permission.

Become a Fellow of BMJ Case Reports today and you can:

- Submit as many cases as you like

- Enjoy fast sympathetic peer review and rapid publication of accepted articles

- Access all the published articles

- Re-use any of the published material for personal use and teaching without further permission

Customer Service

If you have any further queries about your subscription, please contact our customer services team on +44 (0) 2071111105 or via email at support@bmj.com.

Visit casereports.bmj.com for more articles like this and to become a Fellow 